\title{
New Smart City Construction Driven by Innovation
}

\author{
Xiaoli Zhang \\ Shandong Xiehe University, Jinan, Shandong, 250107
}

Keywords: Innovation drive; new type; smart city; construction

\begin{abstract}
From the perspective of technology, this paper discusses the problems in the adjustment of the smart city and the implementation of the development trend. In addition, on the basis of interconnection and intercommunication, this direction of development and innovation is further discussed to provide a theoretical basis for the construction work of the new type of smart city driven by innovation.
\end{abstract}

\section{Introduction}

In the past few years, China's construction work in smart cities has spread to a large number of domestic cities and urban areas. From the perspective of the world, we understand this issue, and the speed of its expansion and the final results are all at the forefront. During this period, during the development of smart cities, whether it is the system of management work, the mechanism during work, and the way in which technology is finally achieved, all through innovation. If there is no innovation, it is tantamount to daydreaming to promote the development of a smart city. Therefore, innovation drive belongs to an important theme that follows the long-term development of smart cities.

\section{The question is raised}

At present, whether it is the leaders in the city, the people involved in the construction of smart cities, or the ordinary people, it is often aware that there are still some loopholes in the current development of smart cities to be optimized, and the results that people hope to achieve are not Realization, after a lot of effort, is very different from the actual expectations. Therefore, leaders, ordinary people and the relevant staff all have great expectations for the development of smart cities. In the face of the current situation, we must continue to drive innovation. However, some of the direction, the foothold and the most important issues during the innovation period are carefully summarized, understood and adjusted.

\section{Adjust the foothold and development trend of smart cities}

In most cities, the trend of smart cities to develop is at least 7 to 10, and in multiple units, it will be promoted in 10 to 20 different fields. There is no problem in this kind of development direction. This is because during the management work, service provision and development of the city, it can play a role through contact with new information technology. However, how can we This type of "wisdom" is used to play a very important role in education, as well as in medical, transportation, and service industries. It is a matter of serious consideration.

China's land area is huge, and the topography and climatic conditions are very different. In the two different regions, medical conditions will face two problems mentioned below: first, public service issues, and second, quality. Problems with sharing medical resources. Based on this, we will explore what can be done during the construction of the two cities. To tell this question is only a unilateral way of thinking about it as a way of thinking about it. At least 7-8, or even about 20 smart cities in all cities in China are developing. After sorting out one by one, find out the problem that is urgently needed to be solved.

For two different cities, the related factors are also different. The existence or non-existence of 
the relevant conditions for solving the problems is also worth exploring. Therefore, it is extremely efficient to do so. Think about things, especially those that are very important and can exert great effects, and then discuss the ideas, techniques, and platforms of development of smart cities. The essence is to solve the problems that arise. In the process, what role can be played out? If the smart city wants to achieve the goal of innovation, the first step is to make innovation in both thinking and business, and then to explore other areas of innovation. Only by having a clear grasp and understanding of the things to be done can the foundation and value of innovation be reflected.

\section{From a technical perspective, discuss the existing problems}

For smart cities, the main part is on the word "wisdom". Therefore, it differs from the previous urban construction work. The most important way is to grasp the data more fully, information and dynamic. Previously, whether it was from medical care, management work in the city, and education, for the transportation field in the city, all such data existed with the business, but the smart city added new things to it. Mainly for the two parts of perception and object. The intelligence of all forms of wisdom has some connection with perception and association. If this part of the connection does not exist, then there is no starting point for wisdom. After the realization of the perception and the integration of the object, there must be a very good transfer function and the function of calculation. The calculation function is compared with the manual processing, the former is more sensitive, the speed is faster, and the work efficiency is also extremely high.

In this process, the existing contradictions mainly arise from the process of promoting different industries. The main contradiction in this link is that there are differences. In some industries, for example, in industries where perceptions and connections are relatively small, and management is much more work, the current interoperability, the ability to analyze data, and the ability to share resources are reduced. However, if it is more intelligent and more effective, then during the connection between perception and the association, it will be understood that the flaw in perception is a major aspect of the existence of contradiction. Taking the industry of healthy pension as an example: At present, the relevant companies and staff of the industry have not formed a mature business model. The main reason is that due to the lack of system, the market is not mature enough for the development of this part of the enterprise [1] .

However, if a new approach to achieving a new healthy pension model is adopted, can the current perceived ability support the needs of the industry? It can be directly pointed out that for the health care industry, the existing perception ability is bound to be insufficient. Support its needs. This is due to the fact that in order to understand in detail the physical conditions of a person's body and activities, there are still huge loopholes in the technology and systems of perception. If there is a lack of perceived ability to support its needs, there can be a high-quality, intelligent type of healthy pension system that will enable the industry to develop rapidly. And this, certainly not the current understanding of the form of medical equipment and other products can be solved.

Is it possible to fully sense during the management of smart types of transportation, medical care, and the city? Nowadays, shared bicycles enter people's sights at a rapid speed and develop, and the precondition that can be realized is the ability to perceive. It is intuitive to understand that if you do not have the ability to perceive, you must not be able to achieve this activity. However, based on the transportation system in a sound city, after integrating the people flow and logistics activities involved, the existing perception ability can really meet this part of the demand [2]. In the case of the current lack of perceived ability, it is a very important technical issue in the development of smart cities. Subdivided, there are two important issues:

First, the ability to perceive most things must be solved in an innovative way, and for sensing technology, it must continue to innovate. The technology itself has a huge space for development. At present, more than $95 \%$ of the innovative research work on sensing technology is in the state of low-level technology, but in terms of innovation, the difficulty is not very large, and the high-end The reason why technology is in the hands of others is because the market size of this industry is extremely small, which is a very important issue. Among universities and professional scientific research departments, the number of institutions with innovative capabilities is huge. This is 
because about $95 \%$ of the technology in sensing technology is not very difficult.

Second, we must closely connect the two systems of perception and business. For smart cities, the most important part of cloud computing, semantic computing, and edge computing, however, is the semantics that exist in computing and perception, as well as the processing of object things. The semantics, the three are effectively connected, and turned into a good organic link, such as a large number of problems in artificial intelligence, is solved in this link [3]. Now, during the construction of smart cities, the starting point mentioned still has some defects, and it needs to produce a new kind of method to deal with the problem, which includes the methods used in processing the data and the capabilities it has. And the ability to be closely related to the entire process of solving the problem, not to explain the relevant technology with abstract theoretical knowledge, due to the differences in the business system itself, during the processing of specific data, There are differences in the quantities, types, time requirements, characteristics, and calculation methods involved. To discuss one thing at a time, to discuss the current level of mastery, whether it is the two fields of transportation and medical care, or other things, to achieve a higher quality, more intelligent level of performance, it is necessary It can only be achieved through slow accumulation and development. Don't link some of the theoretical knowledge with the work to be carried out later. Although it can also be called innovation, this kind of innovation can't solve the actual problems. If you want to solve the problem, you must Internally solve it [4].

\section{The basis of interconnection and intercommunication, further development and innovation}

Take the work carried out by government agencies as an example. Interconnection can closely link information and government responsibilities. This is an extremely important prerequisite. However, in the process of building a smart city, we must use this as a basis for further research. In the case of a dozen or twenty businesses that need to promote work in a smart city, it is probably about two-thirds of the area, significantly exceeding the departmental responsibilities of the government. In most cases, it is with the outside world. Relevant personnel and companies are in close contact. The business characteristics displayed in smart cities have long been far greater than the work content involved in e-government, and must largely exceed e-government.

On the premise of e-government, there is a lot of commonality in interoperability. After the operation of interconnection and interoperability, it can be applied. However, during the development of smart forms of transportation, medical care and water work, unilaterally rely on interconnection. Interoperability does not achieve the desired results. In fact, this link is to be built separately. The independent business units of the government departments are brought together to transform the business involved in water, pension and urban management into an intact business operation. In the case of different institutional business systems, especially in the context of the distribution of the entire society, the process should be constructed [5]. It's not just about building your process, it's about presenting the entire data chain. From a comprehensive point of view, the detailed source of data, the processing of the work, and the guarantee of the interests of all parties, if it is to be clearly expressed, unilaterally relying on the interoperability to carry out work, is far from To the request.

After the interconnection, the above mentioned problems still have certain deficiencies. Therefore, we still have to take a step forward and put the data according to the actual situation, the specific quality, refinement, quantity, formatting and time of the demand for things. Linking together and working in this way can truly achieve innovation. It is necessary to make major innovations in this field. Note that it is not too difficult to carry out innovative research on technology. However, it requires relevant staff to be efficient and forward-looking and highly advanced. If not, it will lead to infrastructure construction. Strong, extremely vulnerable to collapse [6].

\section{Conclusion}

During the innovation and construction of smart cities, technological innovation must be 
regarded as one of the areas, and other aspects must be linked together to achieve innovation. Also, during the construction work for smart cities, the meaning of realizing innovation is based on which types of problems can be solved and what kind of problems can be solved, and the greatest common divisor is found out. The most important point is that in the process of implementing innovation and construction in smart cities, relevant staff must think realistically, adjust their previous footholds, and turn their development goals into the distance, which will inevitably promote the later stages of smart cities. Development is more efficient.

\section{Acknowledgment}

Research project name: Research on smart city construction in shandong province driven by innovation.

2016 Shandong Xiehe University University-level Scientific Research Development Plan Project.

Research project number: XHXY201623

2017 Shandong Province Soft Science Research Project.

Research project number: 2017RKB01267

\section{References}

[1] Dang Anrong, Wang Dan, Liang Jun, et al. Development status and trend of new smart cities in China [J]. Geographic Information World, 2017, 24 (4): 1-7.

[2] Song Yingchang. Wisdom Management and Urban Governance Capability Construction_—Based on the Investigation and Analysis of Four Pilot Areas and Cities in Jiangxi Province[J]. City,2017(1):43-48.

[3] Qiao Guanyu, Xu Weiyue, Lu Dongni. The Path Choice of China's Local Government Governance Modernization in the Construction of Smart Cities_- Taking Nanning City as an Example[J]. Journal of Nanning Vocational and Technical College,2016,21(4):39-43.

[4] Editorial Department of Intelligent Building and Smart City. The 7th China Internet of Things Industry and New Smart City Annual Conference ended successfully [J]. Intelligent Building and Smart City, 2017(1): 24-25.

[5] Lu Wei. Smart and green promotes the integration of innovative new smart city (urban governance) and construction industry big data innovation application exchange conference [J]. China survey and design, 2018 (1).

[6] Feng Lu, Huang Wei, Wu Miaomiao, et al. Promoting the modernization of urban governance with the construction of new smart cities__Ningbo Path Research [J]. Ningbo Economics: Sanjiang Forum, 2016 (9). 